Document downloaded from:

http://hdl.handle.net/10251/56285

This paper must be cited as:

David Gomez-Barquero; Douillard, C.; Moss, P.; Mignone, V. (2014). DVB-NGH: the Next Generation of Digital Broadcast Services to Handheld Devices. IEEE Transactions on Broadcasting. 60(2):246-257. doi:10.1109/TBC.2014.2313073.

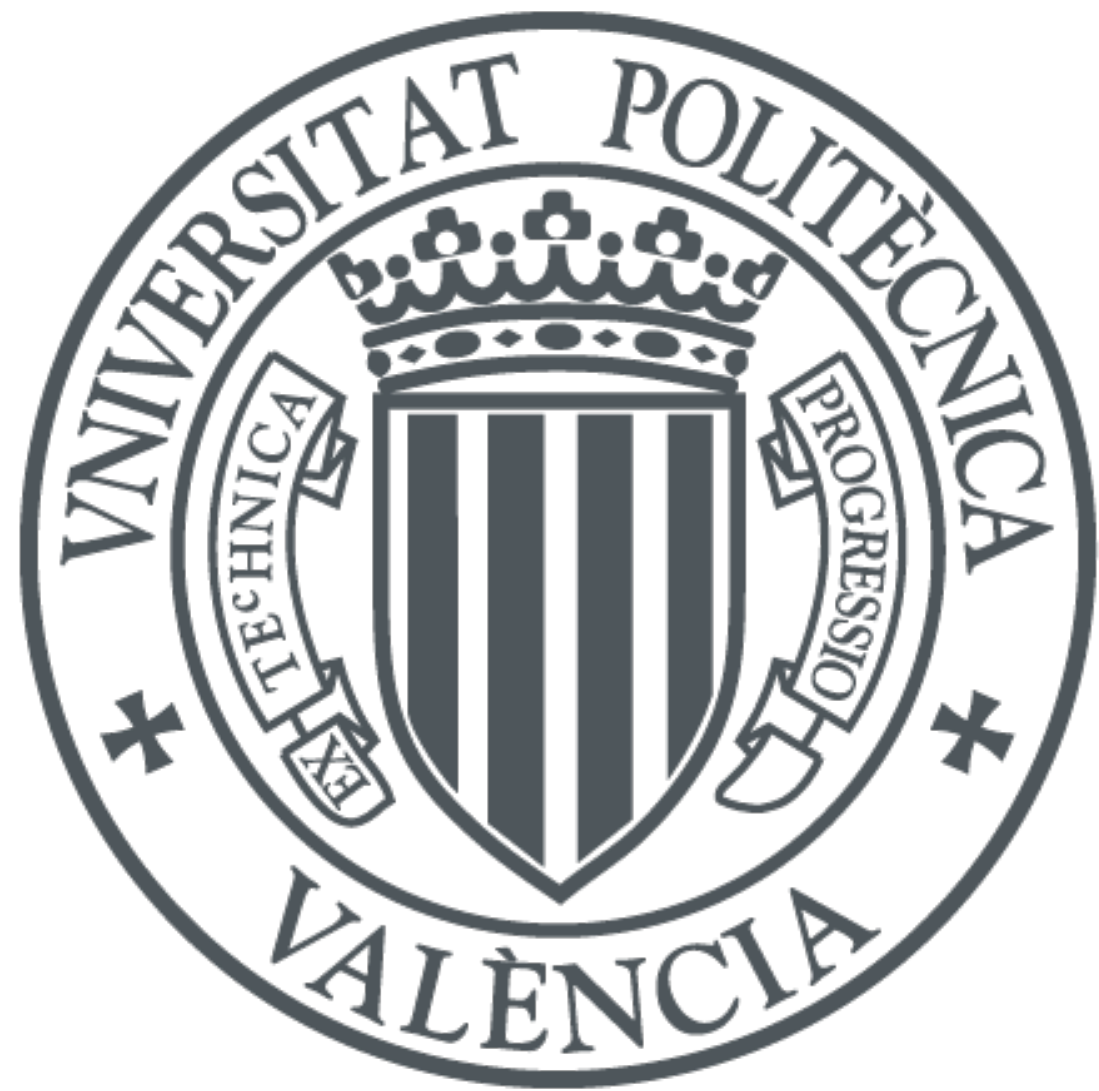

The final publication is available at

http://dx.doi.org/10.1109/TBC.2014.2313073

Copyright Institute of Electrical and Electronics Engineers (IEEE)

Additional Information 


\title{
DVB-NGH: the Next Generation of Digital Broadcast Services to Handheld Devices
}

\author{
David Gómez-Barquero, Catherine Douillard, Peter Moss and Vittoria Mignone
}

\begin{abstract}
This paper reviews the main technical solutions adopted by the next generation mobile broadcasting standard DVB-NGH, the handheld evolution of the second generation digital terrestrial TV standard DVB-T2. The main new technical elements introduced with respect to DVB-T2 are: layered video coding with multiple physical layer pipes, time-frequency slicing, full support of an IP transport layer with a dedicated protocol stack, header compression mechanisms for both IP and MPEG-2 TS packets, new LDPC coding rates for the data path (down to 1/5), non-uniform constellations for 64QAM and 256QAM, fourdimensional rotated constellations for QPSK, improved time interleaving in terms of zapping time, end-to-end latency and memory consumption, improved physical layer signaling in terms of robustness, capacity and overhead, a novel distributed MISO transmit diversity scheme for SFNs, and efficient provisioning of local content in SFNs. All these technological solutions, together with the high performance of DVB-T2, make DVB-NGH a real next-generation mobile multimedia broadcasting technology. In fact, DVB-NGH can be regarded the first third-generation broadcasting system because it allows for the possibility of using multiple input multiple output MIMO antenna schemes to overcome the Shannon limit of single antenna wireless communications. Furthermore, DVB-NGH also allows the deployment of an optional satellite component forming a hybrid terrestrial-satellite network topology to improve the coverage in rural areas where the installation of terrestrial networks could be uneconomical.
\end{abstract}

Index Terms - ATSC 3.0, DVB-NGH, DVB-T2, FoBTV, hybrid terrestrial-satellite, mobile TV, MIMO, MISO, rotated constellations, single carrier OFDM, Time-Frequency Slicing.

\section{INTRODUCTION}

$\mathrm{T}$ HE emergence of smart phones and tablets has renewed the interest on mobile multimedia broadcasting [1]. During the last decade, several mobile broadcast technologies such as DVB-H (Digital Video Broadcasting - Handheld) [2], Media FLO (Forward Link Only) [3] and DVB-SH (Satellite

Manuscript received July 8, 2013; revised January 2, 2014. Parts of this paper have been published in Next Generation Mobile Broadcasting, CRC Press, 2013.

D. Gómez-Barquero is with the iTEAM Research Institute of the Universitat Politècnica de València, Spain (dagobar@iteam.upv.es).

C. Douillard is with Institut Telecom, Telecom Bretagne, France (catherine.douillard@telecom-bretagne.eu).

P. Moss is with British Broadcasting Corporation - Research \& Development (BBC R\&D), United Kingdom (peter.moss@rd.bbc.co.uk).

V. Mignone is with RAI Research and Technical Innovation Centre, Italy (vittoria.mignone@rai.it). to Handheld) [4], were developed to support large scale consumption of mass multimedia services such as mobile television (TV). However, the adoption of mobile TV services did not fulfill the initial expectations due to the lack of a successful business model and the high costs associated to the deployment of new mobile broadcasting networks. Today, a new generation of mobile broadcasting technologies is emerging due to the continuously increasing requirements and expectations of both users and operators, incorporating the latest advances in wireless communications which provide significant capacity and coverage performance improvements compared to first generation mobile broadcast systems.

DVB-NGH (Next Generation Handheld) [5] is the handheld evolution of DVB-T2 (Terrestrial 2nd Generation) [6], the most advanced digital terrestrial TV (DTT) technology in the world, offering more robustness, flexibility and at least 50\% more spectrum efficiency than any other technology [7].

DVB-NGH was created with the objective of becoming the reference mobile multimedia broadcasting standard. However, DVB-NGH not only succeeds significantly outperforming existing mobile broadcasting technologies in terms of capacity and coverage, but also optimizing DVB-T2 in many aspects. Furthermore, DVB-NGH is the first broadcasting system to incorporate Multiple-Input Multiple-Output (MIMO) antenna schemes as the key technology to overcome the Shannon limit of single antenna communications through spatial multiplexing [8], which makes it the first third-generation broadcasting standard.

The standardization process of DVB-NGH started at the beginning of 2010 and finished at the end of 2012. Despite the superior performance compared to existing mobile broadcasting standards, today its commercial success is uncertain and there are no plans for a commercial implementation. But the progress beyond prior state-of-the-art in digital terrestrial broadcasting makes DVB-NGH the reference point for future/upcoming technologies, including not only a potential evolution of DVB-T2 but also for ATSC (Advanced Television Systems Committee), ISDB (Integrated Services Digital Broadcasting), and Future of Broadcast Television Initiative (FoBTV). For example, the MIMO techniques of DVB-NGH provide a starting point for the potential use of MIMO for fixed rooftop reception. Also most of the physical layer proposals for the next-generation TV broadcasting technology ATSC 3.0, currently under evaluation, are based on DVB-T2/NGH. 


\section{A. Analysis of the Commercial Requirements}

The main driver for DVB-NGH was improved coverage to cope with the severe propagation conditions and to reduce the network infrastructure investments. The main commercial requirements of DVB-NGH were [9]:

- The optimization for outdoor and deep indoor portable and slow mobile reception, and also for in-vehicle and outdoor vehicular reception up to $350 \mathrm{~km} / \mathrm{h}$.

- A minimum 50\% spectrum efficiency improvement compared to DVB-H working under the same conditions.

- The possibility of combining DVB-NGH and DVB-T2 signals in one Radio Frequency (RF) channel.

- The possibility of configuring the system either as a unidirectional system, or as a bidirectional system with a return channel provided by a cellular network.

- The minimization of the overhead such as packet headers and metadata without losing any functionality.

- The possibility of offering a service in different qualities with specific robustness and graceful degradation in fringe areas of the network.

- The possibility of transmitting location-based services within Single Frequency Networks (SFN) with a minimum increase to network overhead.

- The possibility of deploying a satellite component as a complement of the terrestrial network.

Regarding performance, DVB-NGH is the first broadcast system to incorporate MIMO to overcome the informationtheoretic limits of Single-Input Single-Output (SISO) wireless communication systems without any additional bandwidth or increased transmit power. The main drawback is that existing DTT network infrastructure (e.g. RF feedings, power combiners and amplifiers, etc.) needs to be upgraded in order to use a second transmit antenna. At the receiver two antennas and RF front-ends are required to demodulate the signal, which also requires a more complex signal processing ${ }^{1}$ [10]. In DVB-NGH a cross-polarization $2 \times 2$ scheme was selected because of the excessive antenna separation required in the UHF band for co-polar antennas and the increased robustness in Line-of-Sight (LoS) conditions. In the standardization process, pioneer work was also performed developing a UHF cross-polar broadcast MIMO channel at a frequency near 500 $\mathrm{MHz}$ based on a channel sounding campaign which was used for performance evaluation [12].

Notwithstanding the superior technical performance of DVB-NGH, one of its main advantages compared to firstgeneration mobile broadcasting DVB systems is the possibility of transmitting DVB-NGH services in-band within a DVB-T2 multiplex in the same RF channel. This feature alleviates the investment required to start providing NGH services, since it is possible to reuse the existing DVB-T2 infrastructure and spectrum without deploying a dedicated

\footnotetext{
${ }^{1}$ Without MIMO, several antennas at the receiver can be used to take advantage of the antenna gain with maximal-ratio combining. Advanced receivers with more than one antenna may also benefit from beamforming signal processing [11].
}

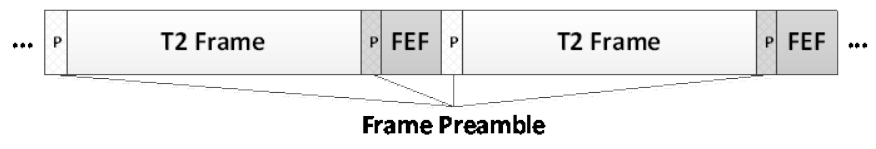

Fig. 1. Co-existence of T2 frames and future extension frames (FEFs) in a single multiplex. Each T2 frame and FEF starts with a preamble OFDM symbol which identifies the type of frame.

DVB-NGH network. This may encourage broadcasters to launch services gradually based on the local market demand.

The combination of DVB-NGH and DVB-T2 in the same multiplex is possible thanks to the Future Extension Frame (FEF) of DVB-T2. FEFs enable to transmit several technologies in the same multiplex in a time division manner. In DVB-T2, all frames start with a preamble OFDM symbol known as P1, which identifies the type of frame. The position in time and the duration of the FEFs are signaled in the physical Layer-1 (L1) signaling in the T2 frames. This way, DVB-T2 receivers not able to decode a FEF simply ignore the transmission. During the transmission of FEFs, terminals can switch-off their RF front-ends saving power, like in a standalone DVB-T2 discontinuous transmission.

Fig. 1 illustrates the combined transmission of DVB-T2 with FEFs in the same multiplex. It should be noted that the technologies transmitted in the FEFs see the T2 frames as "FEFs". The figure shows a typical deployment scenario for DVB-NGH in a shared DVB-T2 multiplex: one FEF of reduced size (e.g. $50 \mathrm{~ms}$ duration) after every $\mathrm{T} 2$ frame with a longer duration (e.g. $200 \mathrm{~ms}$ ). This configuration devotes most of transmission time to T2 services, which are supposed to be the main services for the broadcaster, but it also introduces some mobile services. Moreover, the zapping time of the T2 services is not affected because FEFs are rather short.

Although a FEF can contain any technology, the existence of combined DVB-T2/NGH receivers finally pushed the elaboration of a DVB-NGH physical layer based on DVB-T2.

The combination of DVB-NGH with a cellular network requires an end-to-end IP system in order to deliver the same content over both networks in a bearer agnostic way. It should be pointed out that there is no convergence in networks, since both networks are used separately and independently. Convergence is realized in services, platforms and multi-mode terminals which support both radio access technologies.

DVB-NGH supports two independent transport protocol profiles for MPEG-2 TS (Transport Stream) and IP (Internet Protocol), each one with a dedicated protocol stack. This approach is possible because the physical layer packet unit, known as base band frame (BB frame) and inherited from DVB-T2, is content agnostic. This is an important difference compared to the first-generation DVB standards, whose physical layer units are TS packets, because they additionally require the protocol MPE (Multi-Protocol Encapsulation) to transmit IP. In the second-generation DVB standards, IP can be more efficiently transmitted using the Generic Stream Encapsulation (GSE) protocol. GSE provides efficient IP datagram encapsulation over variable-length link layer packets and can be directly encapsulated into physical layer BB 
frames. The overhead reduction depends on the average size of the IP packets, but savings up to $70 \%$ are feasible.

One of the most important conclusions of the study performed prior to the development of DVB-NGH [13], was that a significant capacity is wasted in DVB-T2 due to signaling overheads such as packet headers and metadata. Hence, an optimization of the signaling and packet encapsulation was recommended. DVB-NGH has considerably improved the bandwidth utilization efficiency without any compromise to the functionality of the system. It has adopted new TS and IP packet header compression mechanisms, and it has reduced the overhead of the L1 signaling and the physical layer adaptation.

The two transport protocol profiles of DVB-NGH were designed for transmitting layered video, such as Scalable Video Coding (SVC), with multiple physical layer pipes $(\mathrm{PLPs})^{2}$. Layered video codecs allow for extracting different video representations from a single bit stream (the different sub-streams are referred to as layers). The combination of a layered video codec with multiple PLPs presents a great potential to achieve a very efficient and flexible provisioning of mobile TV services. By transmitting a base layer using a heavily protected PLP and an enhancement layer in one PLP with moderate/high spectral efficiency, it is possible to costefficiently provide a reduced quality service with a very robust transmission, while providing a standard/high quality for users in good reception conditions [14]. The benefits of using layered video compared to simulcasting the same content with different video qualities in different PLPs with different robustness are twofold. First of all, the bandwidth requirements are reduced, and secondly, it is possible to provide a graceful degradation of the received service quality when suffering strong channel impairments with seamless switching between the different video qualities [15].

The DVB-T2 specification states that DVB-T2 receivers are only expected to decode one single data PLP at a time [6]. DVB-NGH has enhanced the handling of multiple PLPs belonging to the same service being possible to receive up to three data PLPs instead of only one as in DVB-T2.

Regarding local content, the two most important types are news and advertising. The provision of local content in national DTT networks is widely implemented in many countries using temporal windows within the national services. DVB-NGH allows exploring the viability of inserting local content in SFNs in a way that has not been possible before. It allows inserting local content from a single transmitter in the network, or providing local services with the same coverage as the national (global) services.

The NGH study mission [13] also recommended targeting sheer terrestrial networks and hybrid terrestrial-satellite networks in order to avoid market fragmentation, as happened with DVB-H and DVB-SH. A hybrid network is probably the

\footnotetext{
${ }^{2}$ A PLP is a logical channel at the physical layer that may carry one or multiple services, or service components. Each PLP can have different bit rates and error protection parameters (modulation, coding rate, and time interleaving configuration).
}

most cost-effective network topology for mobile broadcasting to wide areas, including not densely populated areas.

The satellite component of DVB-NGH was designed with the goal of keeping the maximal commonality with the terrestrial component to ease its implementation at the receivers. Specific solutions were devised to deal with the particularities of mobile satellite reception and to seamlessly combine the signals coming from the two networks with a single demodulator (tuner). DVB-NGH supports long time interleaving with fast zapping at the physical layer in order to compensate for the long signal outages characteristic of the Land Mobile Satellite (LMS) channel, and Single carrier OFDM (SC-OFDM) to reduce the Peak-to-Average Power Ratio (PAPR) of the satellite signal in order to maximize the efficiency of the high-power amplifier on board the satellite. SC-OFDM was chosen because it provides an important PAPR gain compared to OFDM, although not as high as TDM (Time Division Multiplexing), while preserving many commonalities with OFDM.

Although DVB-NGH technically outperforms DVB-SH, the main advantage is that there is no need to deploy a dedicated terrestrial network in the $\mathrm{S}$ or $\mathrm{L}$ bands. Using FEFs, it is possible to re-use terrestrial infrastructure in the UHF band to provide NGH services in-band of a T2 multiplex, and add the satellite component in the $\mathrm{L}$ or $\mathrm{S}$ frequency bands forming a hybrid Multi Frequency Network (MFN).

\section{B. Standardization Strategy}

The DVB-NGH specification defines four profiles [5]:

1. The base (sheer-terrestrial) profile.

2. The MIMO terrestrial profile.

3. The hybrid terrestrial-satellite profile.

4. The hybrid (terrestrial-satellite) MIMO profile.

The base profile is the only mandatory profile. It is based on the mobile profile of DVB-T2, known as T2-lite ${ }^{3}$ [16]. Indeed, DVB-NGH receivers must support T2-Lite. The base profile of DVB-NGH introduces many technical improvements with respect to DVB-T2:

- More robust FEC (Forward Error Correction) coding rates for the data path down to $1 / 5$.

- Non-uniform constellations for 64QAM and 256QAM.

- Four-dimensional rotated constellation for QPSK.

- Convolutional inter-frame time interleaving.

- Adaptive cell quantization.

- Time-Frequency Slicing (TFS).

- Improved L1 signaling robustness and overhead.

- Dedicated IP and TS protocol stacks.

- Support for layered video with multiple PLPs.

\footnotetext{
${ }^{3}$ T2-Lite was developed to improve the coexistence of fixed and mobile services. It enables to combine in the same multiplex a DVB-T2 signal optimized for high capacity and fixed reception and a T2-Lite signal optimized for high robustness and mobile reception in terms not only of modulation, coding rate and time interleaving, which are possible with multiple PLPs, but also FFT size and pilot pattern. T2-Lite is based on a limited sub-set of the DVB-T2 standard to allow simpler receiver implementations [16].
} 


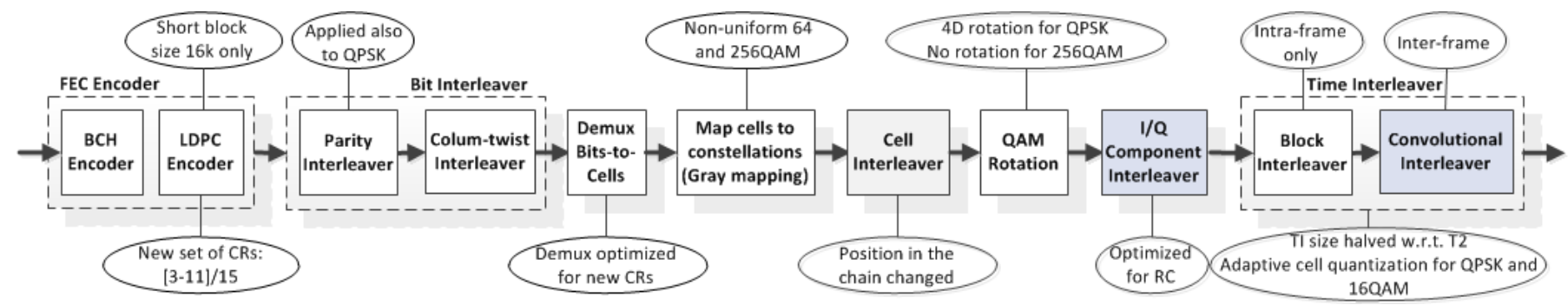

Fig. 2. DVB-NGH BICM module and its differences with DVB-T2. Two new blocks are introduced: I/Q component interleaver and the convolutional time interleaver. The position of the cell interleaver in the chain is changed. As in DVB-T2, the FEC scheme is based on a serial concatenation of a Bose-ChaudhuriHocquenghem (BCH) code and a Low Density Parity Check (LDPC) code. Abbreviations: CRs (Coding Rates). RC (Rotated Constellations).

- IP and TS packet header compression mechanisms.

- Two complementary techniques to efficiently inserting local content in SFNs, known as orthogonal and hierarchical local service insertion.

- A novel distributed MISO (Multiple-Input Single-Output) scheme for SFNs, known as enhanced SFN (eSFN).

The base profile provides full description of all details. The MIMO and hybrid profiles only describe the differences with reference to the base profile. This profile only outlines the differences with respect to the MIMO and hybrid profiles.

The MIMO terrestrial profile defines a 2x2 cross-polarized MIMO system which allows spatial multiplexing of two information data streams across two antennas. Two are the main technical elements introduced:

- A novel MIMO transmission scheme known as enhanced spatial multiplexing with phase hopping (eSM-PH) [17].

- A new bit interleaver that eases the implementation of iterative receivers which can significantly increase the MIMO gain.

The eSM-PH code and the bit interleaver have been also optimized for the case of deliberated power imbalance between the transmit antennas, which may be useful in cases not all receivers support MIMO.

The hybrid profile enables to complement the terrestrial coverage in the UHF band with an optional satellite component in the $\mathrm{L}$ or $\mathrm{S}$ frequency bands. A SFN configuration with both networks operating at the same frequency in the $\mathrm{L}$ or $\mathrm{S}$ bands is also possible. The main technical elements introduced are:

- Long time interleaving (e.g. around 10 seconds) at the physical layer with fast zapping support.

- SC-OFDM for the satellite component in hybrid MFN networks (for hybrid SFN networks, OFDM is used).

The hybrid MIMO profile allows the use of MIMO in the terrestrial and/or the satellite components within a hybrid transmission scenario.

This paper provides an overview of the main technical solutions adopted in DVB-NGH. The paper is structured in four sections, one for each profile. Section 2 describes the base profile. Section 3 is devoted to the MIMO terrestrial profile. Section 4 deals with the hybrid terrestrial-satellite profile. Section 5 describes the hybrid MIMO profile. Finally, the paper is concluded with Section 6 .

\section{BASE PROFILE OF DVB-NGH}

\section{A. Bit Interleaved Coding and Modulation (BICM)}

The BICM module of DVB-NGH is based on T2-Lite with a number of optimizations introduced in order to improve the transmission robustness and increase the spectral efficiency, being at the cutting edge of coded modulation technologies. Fig. 2 shows the BICM module of the base profile of DVBNGH and illustrates the main differences compared to DVBT2. DVB-NGH has the same restrictions adopted in T2-Lite aimed to reduce the receiver complexity, such as only the short LDPC codeword size of 16200 bits (16k) is allowed, the size of the time interleaver memory is halved compared to DVB-T2, and the use of rotated constellations is prohibited in 256QAM [16]. Additionally, the maximum coded data bit rate in DVB-NGH is limited to $12 \mathrm{Mbps}$, including source and parity data (T2-Lite has a restriction of 4 Mbps of the source data).

The BICM modifications introduced to improve the transmission robustness are the following. At FEC level, new more robust LDPC coding rates down to $1 / 5$ were adopted (the most robust coding rate in DVB-T2 is 4/9 and in T2-Lite is 1/3). At the constellation level, non-uniform 64QAM and 256QAM constellations and a 4-dimensional rotated QPSK constellation were introduced. At the interleaving level, the parity bit-interleaver is used for all constellations (not used for QPSK in DVB-T2). The I/Q component interleaving replaces the cyclic Q-delay of DVB-T2 to exploit the signal-space diversity of rotated constellations distributing the components of each rotated symbol with the maximum possible separation in time and frequency.

DVB-NGH optimized the DVB-T2 Time Interleaver (TI) in two aspects. It introduced a Convolutional Interleaver (CI) for inter-frame interleaving (i.e. across multiple frames), keeping the DVB-T2 Block Interleaver (BI) only for intra-frame interleaving, and the use of adaptive cell quantization for making a more efficient use of the TI memory with low order constellations. The utilization of $\mathrm{CI}$ and adaptive cell quantization multiplies by four the maximum interleaving duration compared to T2-Lite with the same TI physical memory. 
The benefits of the CI are that it allows doubling the interleaving depth with the same memory, and that it reduces the average zapping time for the same interleaving depth by about 33\%. These benefits apply only for inter-frame interleaving, when the $\mathrm{CI}$ is used. The adaptive cell quantization technique allows storing twice the number of cells within a given physical time de-interleaving (TDI) memory for QPSK and 16QAM that tolerate a higher quantization noise. This optimization doubles the TI duration for a given service data rate or the service data rate for a given TI duration, for both intra-frame and inter-frame interleaving.

Fig. 3 shows the performance of DVB-NGH and DVB-H in the Rayleigh channel. The curves display the required SNR to achieve a $10^{-4}$ frame error rate for DVB-NGH and $2 \cdot 10^{-4}$ bit error rate after Viterbi for DVB-H. It can be seen that DVBNGH spans a SNR range of more than $20 \mathrm{~dB}$, spanning even negative values, and supports spectral efficiencies ranging from 0.4 to 5.87 bits per constellation symbol (coding rates uniformly distributed over the range $1 / 5$ to $11 / 15$ were adopted). The gap to the Shannon capacity curve ranges from 2 to $3 \mathrm{~dB}$, and the gain compared to DVB-H ranges from 3 to $7 \mathrm{~dB}$. The gain is higher in mobile channels.

\section{B. Time Frequency Slicing (TFS)}

TFS breaks the current paradigm of transmitting broadcast services in a single RF channel to transmit the services across several RF channels with frequency hopping and time-slicing [18]. It was originally proposed in the DVB-T2 standardization process, but was finally made an informative part of the standard (not normative) due to the need of implementing two tuners in the receivers ${ }^{4}$. DVB-NGH adopted TFS for all profiles because it can be operated with a single tuner without adding excessive receiver complexity.

TFS can provide very important gains both in terms of capacity due to enhanced statistical multiplexing (StatMux) and coverage for both fixed and mobile reception due to improved frequency diversity [19]. The combination of many RF channels into a single TFS multiplex allows for an almost ideal StatMux gain for Variable Bit Rate (VBR) services. The frequency diversity can significantly improve the robustness of the transmitted signal, since services can be potentially spread over the whole UHF frequency band.

The main benefit of TFS for DVB-NGH is in terms of improved coverage and transmission robustness. Without TFS, the coverage level at a given location is limited by the RF channel with the lowest signal strength. With TFS, the reception at a particular location is determined by the average signal strength of the RF channels. Hence, an indication of the coverage gain at a particular location may be approximately computed as the difference between the average SNR value of the RF channels and the minimum instantaneous SNR among all RF channels [20], see Fig. 4.

\footnotetext{
${ }^{4}$ During the standardization process, it was found to be impossible to guarantee in all cases a time interval between consecutive frames of the same service long enough for frequency hopping among RF channels with a single tuner.
}

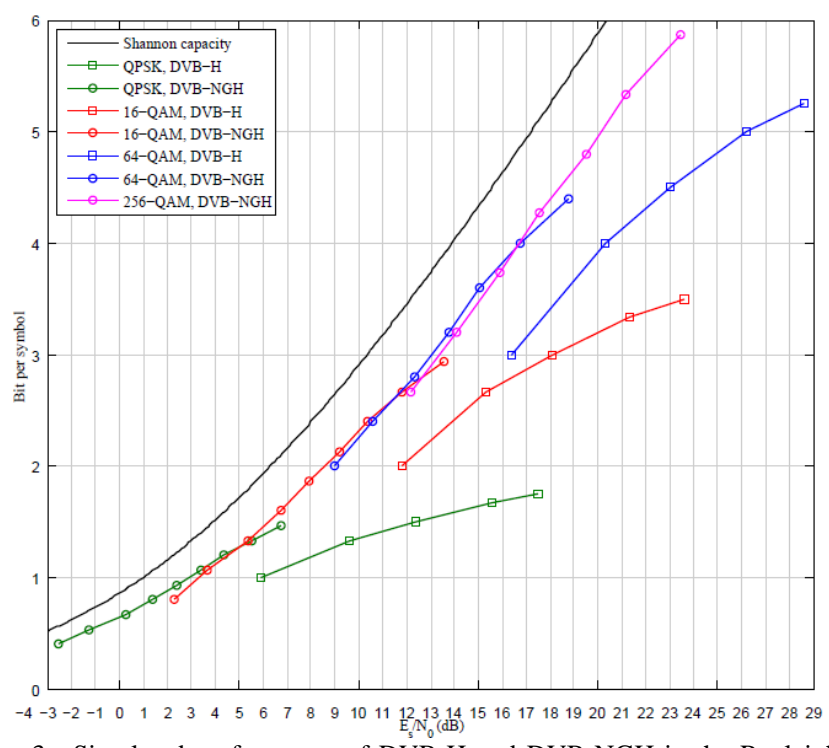

Fig. 3. Simulated performance of DVB-H and DVB-NGH in the Rayleigh fading channel. DVB-NGH 256QAM results are for non-uniform constellations.

In general, the gain increases with the number of RF channels and the frequency spacing. The potential gain is very high because with appropriate coding and interleaving it is possible to cope with a fully lost RF channel. Rotated constellations can further improve the performance by means of increasing diversity [21]. The additional frequency diversity is especially important for pedestrian reception, where very little or non-existent time diversity is available. For mobile reception, the frequency diversity may also reduce the requirements for time interleaving.

Another important benefit of TFS is the possibility to find spectrum more easily and in a more flexible way because it is possible to combine several RF channels with different percentages of utilization allocated to DVB-NGH.

It should be also pointed out that today broadcasting spectrum licenses are for a specific RF channel. Therefore, the implementation of TFS requires new broadcast spectrum regulation approaches to transmit the services across different RF channels.

\section{Logical Frame Structure and FEF Bundling}

The main envisaged use case for DVB-NGH is sharing a multiplex with DVB-T2 using FEFs. For T2-Lite there is a one-to-one relation between FEFs and T2-Lite frames. DVBNGH allows for a more flexible and efficient allocation between FEFs and logical frames. A new logical frame structure has been defined suited to the transmission using FEFs, which has been especially designed to be compatible with TFS, and may be seen as a generalization of TFS. The logical frame structure provides a lot of flexibility, because it relaxes constraints such as having the same length and allocation for the FEFs in all RF channels, or synchronizing the different T2 multiplexes. Furthermore, it allows the combination of FEFs with different transmission modes, frequency bands, etc. enabling hybrid terminals with a single tuner. 


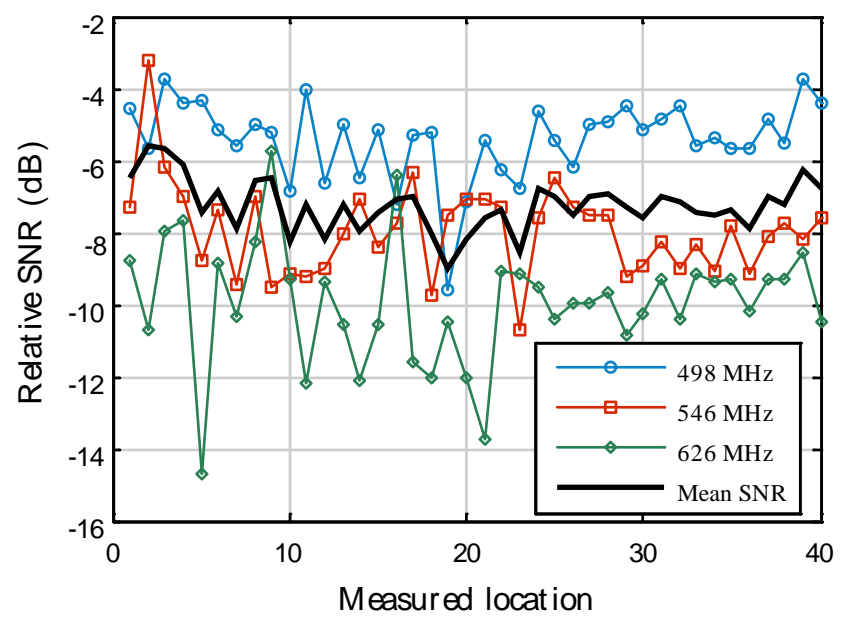

Fig. 4. Samples of the outdoor measurements of three RF channels and average signal level among the three RF channels.

\section{Physical Layer Signaling}

The physical layer signaling of DVB-NGH is transmitted as in DVB-T2 in preamble OFDM symbols at the beginning of each frame. The preamble provides a means for fast signal detection, enabling fast signal scanning, and it carries a limited amount of signaling data transmitted in a robust way that allows accessing the PLPs within the frames.

DVB-NGH has enhanced the physical layer signaling of DVB-T2 in three aspects ${ }^{5}$ :

- Higher signaling capacity.

- Improved transmission robustness.

- Reduced signaling overhead.

The capacity enhancements allow signaling all four profiles of DVB-NGH without any restriction on the number of PLPs used in the system. DVB-NGH has increased the capacity of the signaling preamble and the L1 signaling. The first OFDM symbol of each frame is the preamble P1 symbol. It provides seven signaling bits, which, among other basic information, identifies the type of frame with three bits. These three bits are not sufficient to signal all profiles of DVB-T2, T2-Lite, and DVB-NGH. Hence, DVB-NGH introduced an additional preamble $\mathrm{P} 1$ symbol, known as aP1, to identify the terrestrial MIMO and the hybrid SISO and MIMO profiles. The presence of the aP1 symbol is signaled in the P1 symbol. It should be noted that there is no aP1 symbol for the base profile as in DVB-T2 and T2-Lite. Furthermore, the new logical frame structure of DVB-NGH avoids any limitation in the maximum number of PLPs that can be used due to L1 signaling constraints. The L1 signaling capacity has been increased because it is not constrained to the remaining OFDM symbols of the preamble, known as P2 symbol.

The robustness improvements allow supporting ultra-robust

\footnotetext{
${ }^{5}$ As the physical layer signaling enables the reception of the data, it should naturally be more robust than the data itself. It is generally recommended that the physical layer signaling is $3 \mathrm{~dB}$ more robust than the data [22]. Furthermore, in order to maximize the system capacity, it should introduce as little overhead as possible.
}

modulation and coding rates for the data path such as QPSK 1/5 for both terrestrial and satellite mobile channels and solve the lack of time diversity of the L1 signaling of DVB-T2 [21]. Indeed, the L1 signaling in DVB-NGH can be received at negative SNR under mobility conditions.

DVB-NGH adopts for L1 signaling new mini LDPC codes of size 4320 bits $(4 \mathrm{k})$ with a coding rate $1 / 2$. Although $4 \mathrm{k}$ LDPC codes have a worse performance than the 16k LDPC codes used in DVB-T2 for L1 signaling, the reduced size of 4k LDPC codes is more suitable for L1. In DVB-T2, the LDPC decoding performance for L1 has a degradation because codewords are shortened (i.e. padded with zeroes to fill the information codeword) and punctured (i.e. not all the generated parity bits are transmitted). The robustness gain of 4k LPDC for L1 is around $1 \mathrm{~dB}$ [1]. The adopted 4k LDPC codes have the same parity check matrix structure as the $16 \mathrm{k}$ LDPC codes used for data protection. This allows for efficient hardware implementations at the transmitter and receiver side efficiently sharing the same logic.

DVB-NGH also adopted two new mechanisms to improve the robustness of the L1 signaling known as Incremental Redundancy (IR) and Additional Parity (AP). AP consists of transmitting punctured bits in the previous frame. The IR mechanism extends the original 4k LDPC code into an 8k LDPC code of 8640 bits. The overall coding rate is thus reduced from 1/2 down to 1/4. L1-repetition as in DVB-T2 can be optionally used to further improve the robustness of the L1 signaling as a complement of AP and IR. Overall, the difference between the most robust configurations in DVB-T2 and DVB-NGH is around $4 \mathrm{~dB}$ [1].

The robustness improvement of the L1 signaling can be translated into a reduction of the signaling overhead for the same robustness. But DVB-NGH changed the L1 signaling structure of DVB-T2 in order to reduce the signaling overhead. Instead of signaling the configuration of each PLP, PLPs are associated in groups with the same settings, reducing the required signaling information. Furthermore, it is possible to split in several frames signaling parameters which are transmitted in DVB-T2 in every frame but are static in practice. The new logical frame structure of DVB-NGH also avoids transmitting all L1 signaling information in each frame. The overhead improvements allow reducing the signaling overhead and increasing the system capacity between $1 \%$ and $1.5 \%$ without affecting the system performance [1].

\section{E. System and Upper Layers}

DVB-NGH supports two independent transport protocol stacks for TS and IP, see Fig. 5, which were independently designed to improve the bandwidth utilization. Both protocol stacks also allow the transmission of layered video with multiple PLPs. DVB-NGH allows simultaneously receiving up to three data PLPs plus the common PLP of the same service (DVB-T2 receivers are only capable of receiving one data PLP in addition to the common PLP). A new signaling to map the service components and PLPs was defined together with the scheduling of the PLPs at the physical layer. 


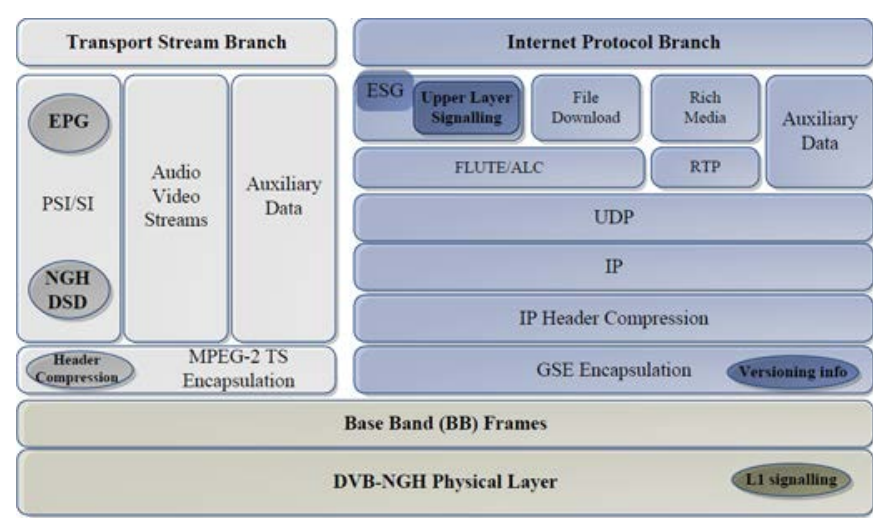

Fig. 5. Transport Stream (TS) and Internet Protocol (IP) profiles of DVBNGH, including optional packet header compression mechanisms [1]. Both profiles may co-exist in the same multiplex.

In Fig. 5, it can be seen that the L1 signaling solution is the same for both stacks. The TS signaling and service discovery is based on the traditional PSI/SI (Program Specific Information/Service Information) tables at the link layer (Layer 2) with a new delivery system descriptor (NGH DSD).

The upper layer solution for IP is based on OMA-BCAST (Open Mobile Alliance Mobile Broadcast Services Enabler Suite), although the specification allows using other solutions. OMA-BCAST is an open global specification for mobile TV and on-demand video services which can be adapted to any IP-based mobile delivery technology. It specifies a variety of features including: content delivery protocols for streaming and file download services, electronic service guide for service discovery, service and content purchase and protection, terminal and service provisioning (e.g. firmware updates), interactivity, notifications, etc.

In order to minimize the signaling redundancy and latency, the OMA-BCAST adaptation for DVB-NGH defined minimal signaling in L2, carrying the whole upper layer signaling, including both service and system signaling information above the IP layer (Layer 3) inside the OMA-BCAST service guide structures (e.g. Electronic Service Guide, ESG).The encapsulation of the IP packets at the link layer is done using the GSE protocol and in accordance with the GSE link layer control specification.

\section{F. Overhead Reduction Methods}

DVB-NGH reduces the overhead of DVB-T2 at the physical layer thanks to its improved signaling and physical layer adaptation, as well as at the network layer through the introduction of TS and IP packet header compression.

The improved physical layer adaptation reduces the size of the $\mathrm{BB}$ frame header from 8 bytes down to 3 bytes. The signaling was reorganized separating the PLP-specific information (only present in the L1 signaling) from the BB frame information (only present in the BB frame headers), avoiding duplication and improving consistency. The L1 signaling overhead saving increases the system capacity between $1 \%$ and $1.5 \%$.

The novel TS packet header compression method adopted

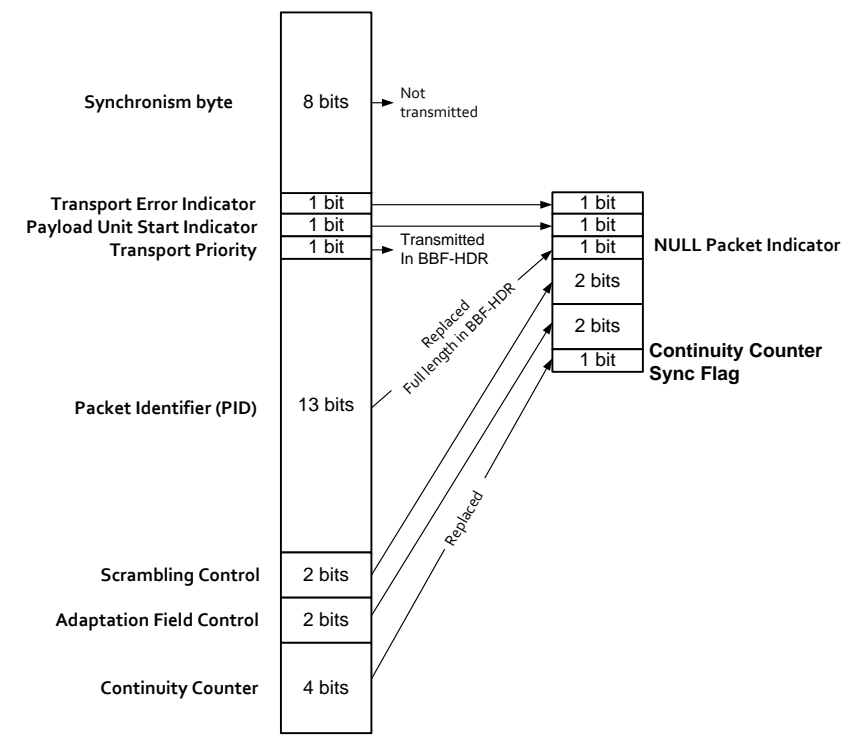

Fig. 6. MPEG-2 Transport Stream (TS) packet header compression [1].

in DVB-NGH reduces the header size from 4 bytes down to only 1 byte, providing $1.1 \%$ system capacity increase. The compression is performed on the transmitter side and the information needed for restoring the header in the receiver is signaled in the BB frame header and the L1 signaling, such that the compression and decompression process is transparent. This technique is applicable only to PLPs that carry one program component. The TS packet header is compressed as follows, see Fig. 6:

- The synchronism byte is removed as in DVB-T2.

- The 1-bit transport priority indicator is removed and transmitted in the BB frame header.

- The 13-bit Program ID (PID) field is replaced by a single bit to signal null packets. The PID value is signaled in the BB frame header.

- The 4-bit continuity counter is replaced with a 1-bit duplication indicator.

The IP packet header compression method adopted in DVB-NGH is based on the unidirectional mode of the Robust Header Compression (ROHC) protocol [23]. ROHC introduces inter-packet dependencies in the transmitted stream which can increase the zapping time and introduce packet error propagations. Hence, a NGH adaptation layer was introduced to diminish the increase in the zapping time and to improve the robustness of the compressed flow, see Fig. 7. The adaptation layer is backwards-compatible with the standalone ROHC framework, which allows reusing existing software implementations of the ROHC protocol.

ROHC can be modeled as an interaction between two state machines, one compressor machine and one decompressor machine. The ROHC framework defines the state machine transitions and describes procedures for starting the transmission and error recovery. ROHC classifies the protocol headers fields depending on their changing pattern between consecutive packets into three types: inferred, static and dynamic. The inferred fields are the ones that contain values 


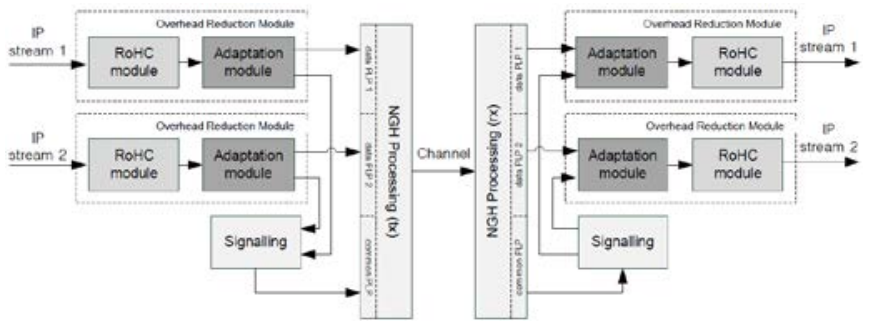

Fig. 7. Block diagram of DVB-NGH including IP header compression [1].

which can be inferred from other protocol header fields or from lower-level protocols and do not need to be transmitted. The static fields are expected to be constant throughout the lifetime of the packet flow (e.g. IP destination address) and therefore must be communicated to the receiver only once, or expected to have well-known values (e.g. IP version) and therefore do not need to be communicated at all. The dynamic fields are the ones that vary during the transmission of the packet flow. The efficiency of the ROHC scheme depends on the setup of the compressor, the characteristic of the transmitted IP flow, and whether or not the NGH adaptation layer for ROHC is used. But with ROHC the IP packet overhead can be reduced to approximately $1 \%$ of the transmitted data, yielding a capacity increase between $2.5 \%$ and $3.5 \%$ [1].

\section{G. Local Service Insertion in Single Frequency Networks}

DVB-NGH adopted two complementary techniques to transmit local content in SFNs, known as hierarchical and orthogonal local service insertion (H-LSI and O-LSI, respectively). Both techniques provide very important capacity gains compared to the classical SFN approach where the local content is transmitted in the whole network, but each technique addresses different use cases with different coverage-capacity performance trade-off, such that the optimum transmission technique depends on the target use case and the particular scenario considered (location and power of the transmitters, distribution of the local service areas, etc.). For both techniques, the transmission of local content through the whole SFN network can be scheduled in a way that different local areas do not interfere with each other.

H-LSI uses hierarchical modulation [24], which allows combining two independent data streams into a single stream with different robustness. With H-LSI, local services are transmitted in a Low Priority (LP) stream on top of the global services in a High Priority (HP) stream. Transmitters insert local content adding an additional QPSK constellation on top of the constellation used for global services, which can be QPSK or 16QAM. An illustrative example is shown in Fig. 8. When the global PLP employs QPSK and the same coding rate is used for the global and local PLPs, it is possible to double the capacity when all OFDM sub-carriers are used to transmit local content. The maximum percentage of local services in this case is $50 \%$.

H-LSI can be used to transmit local services in areas close

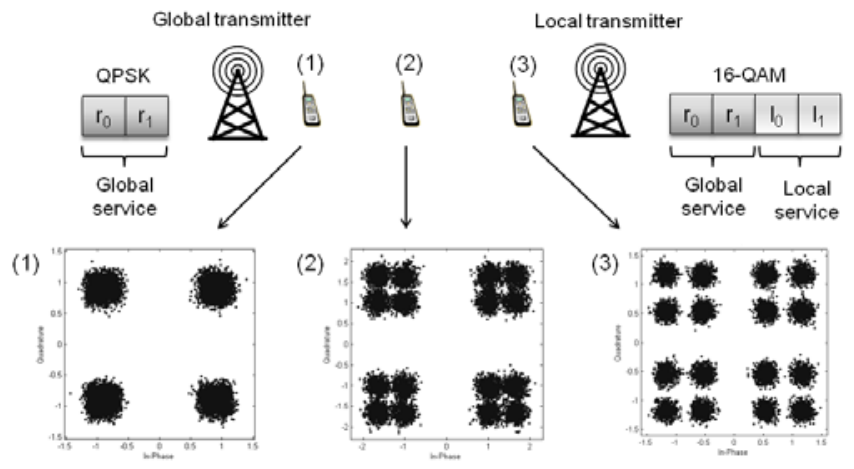

Fig. 8. Illustrative example of H-LSI. Global services are transmitted using QPSK. The transmitter on the right inserts local content transmitting a hierarchically modulated 16QAM. The constellation diagrams show the received signal in different locations as a function of the distance to the local transmitter [1]

to the transmitters, but in some areas it is not possible to receive any local service at all. Another drawback is that the hierarchical modulation suffers from inter-layer interference, because each stream acts as noise with respect to the other, which reduces the coverage of the global services when local services are transmitted. This degradation can be reduced by increasing the spacing between HP constellation symbols or using a lower coding rate, at the expense of degrading the performance of the LP stream or reducing the capacity for global services, respectively.

O-LSI defines groups of OFDM sub-carriers in specific OFDM symbols for the exclusive use of particular transmitters to transmit local services. For each local transmitter, only some sub-carriers within such OFDM symbols are active. The main benefits are twofold. Firstly, the coverage of the global services is not affected by the local services. Secondly, the coverage of the local services is very similar to the coverage of the global services. Local services do not fully benefit from the SFN gain (except within a local service area containing several transmitters). Hence, there is no power gain but there is a statistical network gain. In the overlapping zones between adjacent transmitters, receivers can decode more than one local service in addition to the global service because services do not interfere with each other.

With O-LSI the transmission capacity for local services can be increased using a transmission mode with a higher spectral efficiency than the mode used for global services because it is possible to transmit the OFDM sub-carriers devoted to local services with higher power. The transmission power remains constant, but in the OFDM symbols where local content is transmitted each transmitter has only one set of local subcarriers is active. The capacity gain depends on the percentage of local services and the number of local service areas, but it can also reach values up to $100 \%$ [1].

\section{H. Enhanced Single Frequency Network MISO scheme}

DVB-NGH adopted the distributed MISO scheme of DVBT2 based on Alamouti coding [25], and a novel scheme known as eSFN. They are applied across several transmitters in SFNs reusing the existing DTT network infrastructure and 


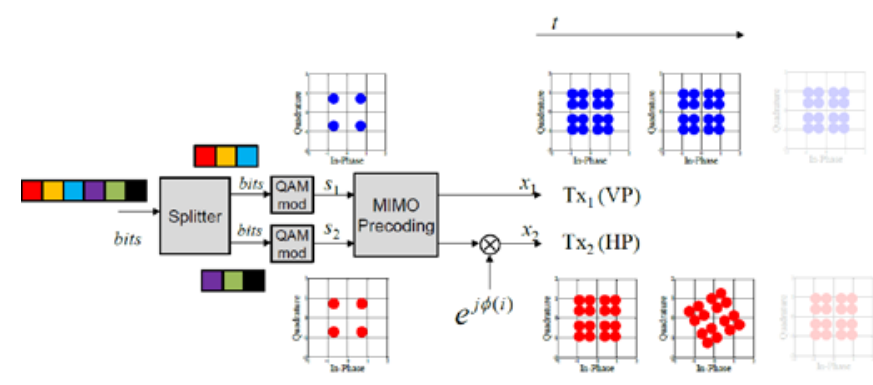

Fig. 9. Block diagram of eSM-PH with QPSK on each antenna [1].

being compatible with single antenna receivers. The Alamouti code is applied across pairs of transmitters, whereas eSFN can be applied to multiple transmitters.

eSFN is a cyclic delay diversity [26] scheme which consists in applying a linear predistortion function to each antenna in such a way that it does not affect the channel estimation in receivers. It increases the frequency diversity of the channel without the need of specific pilot patterns or signal processing to demodulate the signal. This is an advantage with respect to the Alamouti code, because it requires doubling the number of pilot sub-carriers such that receivers can estimate the channel response from each transmit antenna. The randomization performed by eSFN in each transmitter can avoid the negative effects caused by LoS components in SFNs. eSFN may also be used for transmitter identification by using a different predistortion function for each transmitter.

\section{MIMO TERRESTRIAL PROFILE OF DVB-NGH}

DVB-NGH defines the implementation of a $2 \times 2$ crosspolarized MIMO system as an optional profile in order to exploit the diversity and capacity advantages made possible by the use of multiple transmission elements at the transmitter and receiver. The MIMO scheme is known as enhanced spatial multiplexing with phase hoping $(\mathrm{eSM}+\mathrm{PH})$. The use of the MIMO profile is signaled in preamble P1 symbol, which is followed by an additional preamble aP1 symbol that provides information about the FFT size and guard interval used.

eSM-PH retains the multiplexing capabilities of spatial multiplexing and increases the robustness against spatial correlation preventing frequency independent interaction between co-polarized and cross-polarized LoS components. The conceptual block diagram of eSM-PH is illustrated in Fig. 9. The transmission matrix of eSM can be represented as the concatenation of the regular SM transmission matrix with a precoding matrix such that the information symbols are weighted and combined before their transmission across the antennas. The weighting of the information symbols depends on a rotation angle, which was optimized for each constellation. In addition, a phase hopping term was added to the second antenna in order to randomize the code structure and avoid the negative effect of certain channel realizations. The phase hopping term changes periodically within each FEC codeword.

Fig. 10 compares the performance of eSM-PH with SISO,

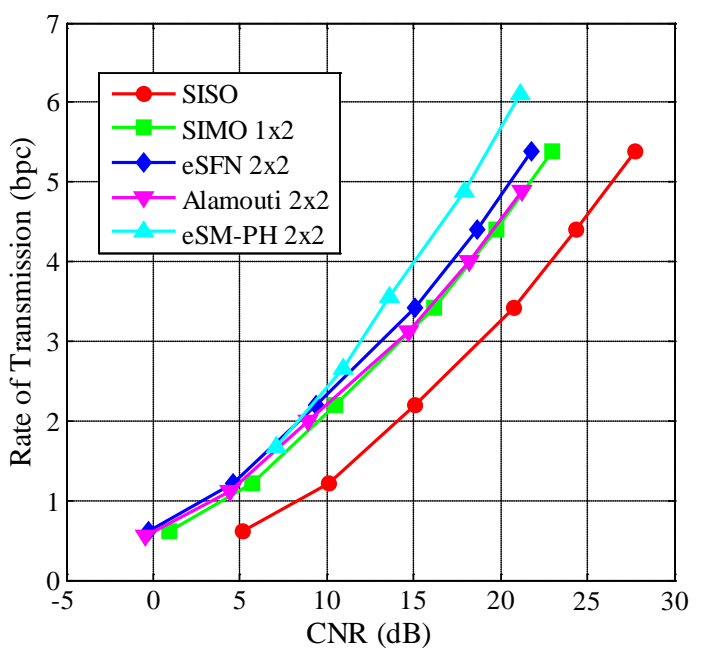

Fig. 10. Performance of the different DVB-NGH MIMO schemes in the NGH Outdoor MIMO channel with $60 \mathrm{~km} / \mathrm{h}$ speed [1].

SIMO with two receive antennas, and eSFN and Alamouti with two transmit and two receive antennas. The results include the effect of pilot overhead. A pilot density of $1 / 12$ has been assumed for SISO, SIMO and eSFN, whereas for Alamouti and eSM-PH the pilot density is 1/6 (i.e. double, the pilot patterns used for eSM-PH are the same as for MISO Alamouti in DVB-T2).

The figure highlights that when the pilot overhead is taken into account most of the gain achieved by the MIMO schemes over SISO comes from having a second receiving antenna. Indeed, the performance gain of MIMO Alamouti is practically compensated due to the effect of increased pilot overhead. eSFN outperforms MIMO Alamouti because it does not require to double the pilot overhead. Compared with SISO and with $15 \mathrm{~dB}$ of average CNR, SIMO provides almost a $50 \%$ capacity increase, or equivalently around $4.5 \mathrm{~dB}$ of CNR gain. eSM-PH provides interesting gains only in favorable reception conditions, that is, for high CNRs, which can be achieved for the portable outdoor or vehicular reception use cases envisaged for DVB-NGH. However, the potential benefit of MIMO is greater for rooftop reception due to the higher signal levels available to a rooftop antenna, where CNRs over $20 \mathrm{~dB}$ are realistic, because the gain increases with the CNR. At this value, the MIMO capacity gain is around $66 \%$ over SISO and $20 \%$ over SIMO.

Furthermore, it should be pointed out that the MIMO profile has also adopted a new bit interleaver that exploits the quasi-cyclic structure of the adopted LDPC codes. It exhibits a low complexity, low latency, and fully parallel design that ease the implementation of iterative structures which can provide significant gains (around $1 \mathrm{~dB}$ ) on the top of the MIMO gain [17].

eSM-PH can be transmitted with power imbalance between the antennas to ease its introduction and co-existence with SISO transmissions. A deliberate transmitted power imbalance avoids envelope power fluctuations at the transmitter at the cost of a small, probably acceptable coverage reduction for SISO/SIMO terminals by lowering the existing transmit 


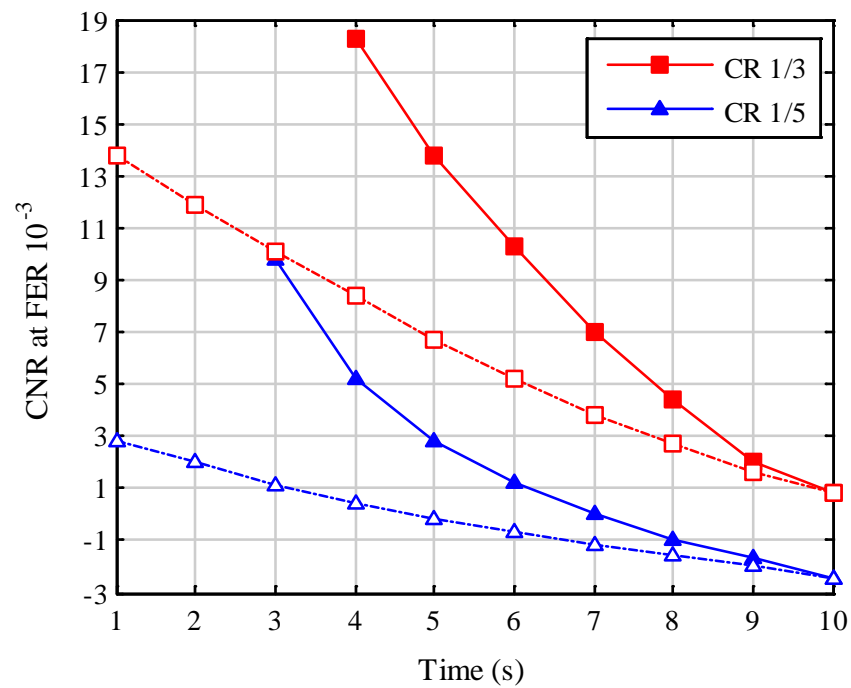

Fig. 11. Performance during the transitory period from fast access decoding to decoding of the complete codeword. Continuous lines correspond to uniform and dashed lines correspond to uniform-late profile with $50 \%$ of information in the late part. TU6 channel with $33 \mathrm{~Hz}$ Doppler [1].

antenna power slightly, while eSM-PH maintains good performance due to its optimized performance to overcome this situation. The power imbalances considered in the standard are $3 \mathrm{~dB}$ and $6 \mathrm{~dB}$. For these cases, the eSM-PH rotation angle has been optimized to reduce the performance loss due to transmit power imbalance [17].

\section{Hybrid Terrestrial-Satellite Profile of DVB-NGH}

DVB-NGH allows for the deployment of an optional satellite component complementing the coverage provided by a terrestrial network. The hybrid profile specifies the use of extended convolutional inter-frame time interleaving with fast zapping support with a uniform-late CI profile, SC-OFDM to reduce the PAPR of the satellite transmitted signal in hybrid MFNs, and the scheduling of the terrestrial and satellite transmissions such that parallel reception of both signals is possible for terminals with a single tuner in hybrid MFNs. This has been made possible thanks to the introduction of the concept of logical channel and logical channel group [1].

The hybrid profile makes use of an external TDI memory as a complement of the on-chip TDI memory used in the base terrestrial profile. The on-chip TDI memory is only used for intra-frame block interleaving, whereas the external TDI memory is used for inter-frame convolutional interleaving. Compared to a sheer terrestrial receiver, a hybrid receiver requires at least an additional external time de-interleaving memory to account for the long time interleaving requirements at the physical layer, SC-OFDM demodulation, and a tuner covering the satellite frequency bands ( $\mathrm{L}$ and $\mathrm{S}$ ).

Fast zapping is supported using a uniform-late profile of the CI, like in DVB-SH [27]. Generally, it is considered that zapping times around one second are satisfactory, whereas more than two seconds are felt as annoying. The uniform-late profile introduces a trade-off between overall performance in

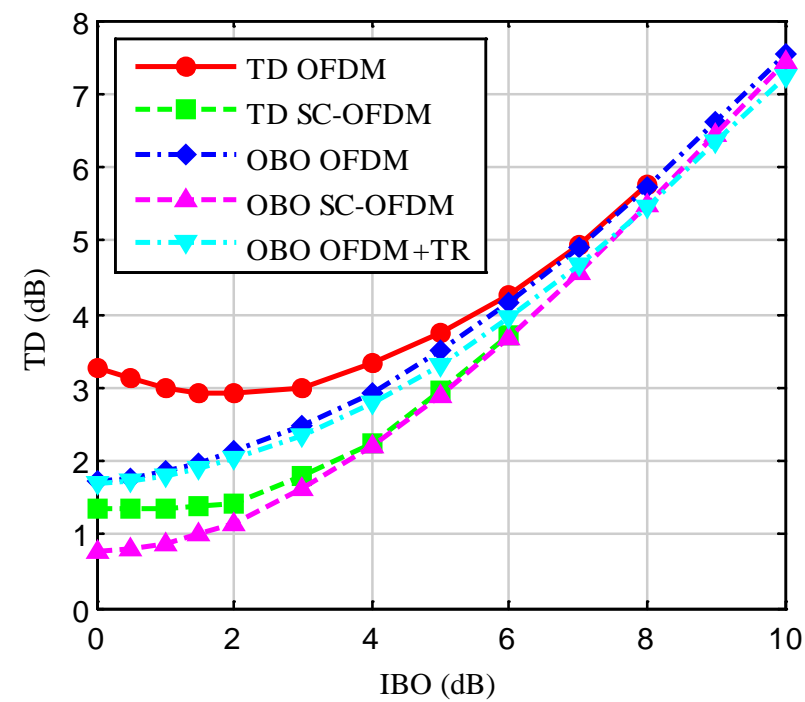

Fig. 12. Total Degradation (TD) and Output Back-Off (OBO) performance as a function of the Input Back-Off (IBO) of OFDM and SC-OFDM in a linearized TWTA power amplifier, AWGN channel [1].

mobile channels and performance after zapping. The larger the size of the late part (i.e. proportion of the LDPC codewords transmitted in one or multiple late frames with a duration of typically less than $1 \mathrm{~s}$ ), the better the performance after zapping. The drawback is that the overall performance in mobile channels is reduced because it results in a non-uniform interleaving of information over time.

Figure 11 shows an illustrative example of the performance over time with the uniform and uniform-late $50 \%$ CI profiles with a TI duration of $10 \mathrm{~s}$. A time-sliced transmission has been assumed, with a cycle time of $1 \mathrm{~s}$. It can be seen that the uniform-late CI profile provides fast zapping for users in good reception conditions (the lower the coding rate, the better the zapping performance), whereas for the uniform CI profile users need to receive several bursts to start reproducing the service. For the considered channel model, after $10 \mathrm{~s}$ the performance of both profiles is identical. This is not the case for the LMS channel, where the uniform-late profile shows a performance degradation between 1 and $2 \mathrm{~dB}$ [1].

SC-OFDM provides an approximate gain with respect to OFDM in the order of $2.5 \mathrm{~dB}$ in terms of reduced PAPR for high-power satellite amplifiers, which can be directly translated into an increase of the coverage provided by the satellite, achieving a gain of about $1.5 \mathrm{~dB}$ in the link budget at low input back-offs, see Fig. 12. In the figure, the optimum input back-off for OFDM is $2 \mathrm{~dB}$ which yields a total degradation of $2.95 \mathrm{~dB}$, whereas the optimum input back-off for SC-OFDM is $1 \mathrm{~dB}$ leading to a total degradation (TD) of $1.35 \mathrm{~dB}$. SC-OFDM enables operating closer to the saturation point than the OFDM, thus improving the power efficiency of the amplifier.

From an implementation point of view, the main difference at the receiver with respect the base OFDM profile is the introduction of a de-spreading function (i.e. an additional iFFT). The spreading at the transmitter can be viewed as a 
way of spreading each symbol over the entire spectrum. Hence, the implementation cost of including SC-OFDM in the chips is markedly lower than that of TDM modulation.

The use of the hybrid profile is signaled in preamble P1 symbol, which is followed by an additional preamble aP1 symbol that provides information about the use of SC-OFDM, and the used FFT size and guard interval.

\section{HYBRID MIMO PROFILE OF DVB-NGH}

The hybrid MIMO profile allows the use of MIMO on the terrestrial and/or satellite elements within a hybrid terrestrialsatellite network. Cases included are one or two (crosspolarization, linear polarization) terrestrial antennas in combination with one or two (cross-polarization, counterrotating circular polarization) satellite antennas, making it possible to use up to four transmit antennas. At least one of the transmission elements (i.e. terrestrial or satellite) must employ multiple antennas; otherwise, the use case lies within the hybrid profile. Both MFN and SFN network configurations are possible.

For hybrid MFN configurations, in the case that satellite waveform is SC-OFDM, spatial multiplexing encoding for rate-2 MIMO is simple spatial multiplexing instead of eSM$\mathrm{PH}$ (i.e. no MIMO precoding nor phase hopping is applied).

For hybrid SFN configurations, the satellite simply repeats the transmission of the terrestrial transmitters to avoid increasing the pilot overhead required for channel estimation (e.g. in the four-transmitter case, if channel responses for all channels are required the pilot density is four times the density required for the same estimation accuracy to the one transmitter SISO case). Another consideration motivating this choice is the need to guarantee a satisfactory receiver performance is possible even if one of the streams is lost (a highly probable event in such mobile systems). The selected codes for SFN scenarios are presented in Table 1.

When single polarization is available at both satellite and terrestrial site, Alamouti coding or eSFN can be applied. When the terrestrial transmissions are dual polarized and the satellite utilizes only a single polarization, the satellite can simply transmit the same symbols as one terrestrial polarization (this is called "Alamouti + QAM"). eSFN can also be used.

To achieve a rate-2 transmission, Virtual MIMO (VMIMO) can be used, where the single polarized transmitter emulates at the transmitter side an optimized $2 \times 1$ channel while the dual polarized transmitter emits rate-2 MIMO. However, VMIMO is generally not practical on the satellite side due to its high SNR requirement. When the terrestrial transmissions use single polarization and satellite transmissions are dual polarized, Alamouti + QAM can be utilized. Now only the roles of terrestrial and satellite transmitters are reversed as compared to the code presented for the case of dual terrestrial and single satellite polarization. eSFN and VMIMO are also possible. In the final case both terrestrial and satellite transmissions utilize dual polarization. For rate- 1 the same
TABLE I

MIMO SCHEMES FOR DIFFERENT HYBRID MIMO SFN SCENARIOS.

\begin{tabular}{|c|c|c|c|}
\hline $\begin{array}{l}\text { Number } \\
\text { of trxs. }\end{array}$ & Terrestrial & Satellite & Schemes \\
\hline 2 & $\begin{array}{c}\text { Single } \\
\text { Polarization }\end{array}$ & $\begin{array}{c}\text { Single } \\
\text { polarization }\end{array}$ & Rate-1: eSFN, Alamouti \\
\hline 3 & $\begin{array}{c}\text { Dual } \\
\text { Polarization }\end{array}$ & $\begin{array}{c}\text { Single } \\
\text { polarization }\end{array}$ & $\begin{array}{c}\text { Rate-1: eSFN, } \\
\text { Alamouti+QAM } \\
\text { Rate-2: VMIMO }\end{array}$ \\
\hline 3 & $\begin{array}{c}\text { Single } \\
\text { Polarization }\end{array}$ & $\begin{array}{c}\text { Dual } \\
\text { polarization }\end{array}$ & $\begin{array}{c}\text { Rate-1: eSFN, } \\
\text { Alamouti+QAM } \\
\text { Rate-2: VMIMO }\end{array}$ \\
\hline 4 & $\begin{array}{c}\text { Dual } \\
\text { polarization }\end{array}$ & $\begin{array}{c}\text { Dual } \\
\text { polarization }\end{array}$ & $\begin{array}{c}\text { Rate-1: eSFN, Alamouti + } \\
\text { Alamouti } \\
\text { Rate-2: eSM-PH (terr.), } \\
\text { eSM-PH + eSFN (sat.) }\end{array}$ \\
\hline
\end{tabular}

Alamouti blocks are transmitted from both satellite and terrestrial transmitters. Further, eSFN can be used for satellite to enhance the operation. This code is called "Alamouti + Alamouti”. For rate-2, eSM-PH is used in both terrestrial and satellite components. The satellite transmits the same signal except for the eSFN predistortion.

The use of the hybrid MIMO profile is signaled in preamble $\mathrm{P} 1$ symbol, which is followed by an additional preamble aP1 symbol that provides information about the use of SC-OFDM, and the FFT size and guard interval used. The hybrid MIMO profile allows combining long time interleaving with MIMO [28].

\section{CONCLUSIONS}

DVB-NGH is the first third-generation DVB standard to include MIMO antenna techniques. It is also the first mobile broadcast system that exploits the diversity of the mobile channel in all dimensions: frequency diversity among several RF channels with TFS, time diversity with long time interleaving (in the order of $10 \mathrm{~s}$ ) at the physical layer with fast zapping support, and space diversity with MIMO. Furthermore, two-dimensional and four-dimensional rotated constellations can be used to increase the diversity order (signal-space diversity). All the new technological solutions adopted in DVB-NGH, together with the high performance of DVB-T2, make DVB-NGH a technology with superior capacity and coverage performance compared to existing mobile multimedia broadcasting standards.

DVB-NGH could also take advantage of the introduction of the new High-Efficiency Video Coding (HEVC) standard, which provides significantly improved coding efficiency as compared with the current state-of-the-art MPEG-4 AVC video coding. However, questions remain regarding the commercial prospects of the standard given the limited success of similar standards in the past. Notwithstanding the superior technical performance of DVB-NGH, one of its main advantages compared to first-generation mobile broadcasting DVB systems is the possibility of transmitting DVB-NGH services in-band within a T2 multiplex in the same RF channel without constraints from coexistence, enabling to reuse the existing DVB-T2 infrastructure and avoiding to deploy a 
dedicated DVB-NGH network. However, the mobile profile of DVB-T2 known as T2-Lite also has this feature. In this respect, the hybrid terrestrial-satellite profile of DVB-NGH may be the one with a higher commercial potential initially, and the terrestrial profile could perhaps reach the market as an evolution of T2-Lite if it becomes successful.

But in any case, progress beyond prior state-of-the-art achieved with DVB-NGH and the pioneering work on the use of MIMO in digital terrestrial broadcasting makes it the reference point for future/upcoming technologies including not only a potential evolution of DVB-T2 but also for ATSC, ISDB, and FoBTV. Indeed, most of the physical layer proposals for the next-generation TV broadcasting technology ATSC 3.0, currently under evaluation, are based on DVBT2/NGH.

\section{ACKNOWLEDGMENT}

The authors would like to thank all the colleagues from the DVB TM-H working group that contributed to the development of the DVB-NGH specification.

\section{REFERENCES}

[1] D. Gómez-Barquero (ed.), "Next Generation Mobile Broadcasting," CRC Press, 2013.

[2] G. Faria, J. A. Henriksson, E. Stare, and P. Talmola, "DVB-H: Digital Broadcast Services to Handheld Devices," Proc. of the IEEE, vol. 94, no. 1, pp. 194-209, 2006.

[3] M. R. Chari et al., "FLO physical layer: An overview," IEEE Trans. on Broadcasting, vol. 52, no. 1, pp. 145-160, 2007.

[4] I. Andrikopoulos, et al., "An Overview of Digital Video Broadcasting via Satellite Services to Handhelds (DVB-SH) Technology," Handbook of Mobile Broadcasting: DVB-H, DMB, ISDB-T and MediaFLO, CRC Press, 2008.

[5] DVB Document A160, "Digital Video Broadcasting (DVB); Next Generation Broadcasting System to Handheld, Physical Layer Specification (DVB-NGH),” Nov. 2012.

[6] L. Vangelista, et al., "Key Technologies for Next-Generation Terrestrial Digital Television Standard DVB-T2," IEEE Communications Magazine, vol. 47, no. 10, p. 146-153, 2009.

[7] I. Eizmendi, G. Prieto, G. Berjon-Eriz, I. Landa, and M. Velez, “Empirical DVB-T2 Thresholds for Fixed Reception," IEEE Trans. on Broadcasting, vol. 59, no. 2, pp. 306-316, 2013.

[8] J. Paulraj, D. A. Gore, R. U. Nabar, and H. Bölcskei, "An Overview of MIMO Communications - A Key to Gigabit Wireless," Proc. of the IEEE, vol. 92, no. 2, pp. 198-218, 2004.

[9] DVB Commercial Module sub-group on Next Generation Handheld, “Commercial Requirements for DVB-NGH,” CM-1062R2, June 2009.

[10] I. Sobron, et al., "Low-Complexity Detection of Full-Rate SFBC in BICM-OFDM Systems," IEEE Trans. on Communications, vol. 60, no. 3, pp. 626-631, 2012.

[11] L. Yang, G. Ren, W. Zhai, and Z. Qiu, "Beamforming Based Receiver Scheme for DVB-T2 System in High Speed Train Environment,” IEEE Trans. on Broadcasting, vol. 59, no. 1, pp. 146-154, 2013.

[12] P. Moss, T. Y. Poon, and J. Boye, "A Simple Model of the UHF CrossPolar Terrestrial Channel for DVB-NGH,” BBC White Paper, 2011.

[13] DVB Technical Module sub-ground on Next Generation Handheld, "NGH Study Mission Report," TM-H 411, June 2008.

[14] C. Hellge, T. Wiegand, E. Guinea Torre, D. Gómez-Barquero, and T. Schierl, "Efficient HDTV and 3DTV Services over DVB-T2 Using Multiple PLPs with Layered Media," IEEE Communications Magazine, vol. 57, no. 7, pp. 130-137, 2013.

[15] A. Ksentini and T. Taleb, "QoE-Oriented Adaptive SVC Decoding in DVB-T2,” IEEE Trans. on Broadcasting, vol. 59, no. 2, pp. 251-264, 2013.
[16] D. Gozálvez, D. Gómez-Barquero, I. Eizmendi, G. Berjón-Eriz, and M. Vélez, "DVB-T2 for Mobile and Mobile DVB-T2 (T2-Lite)," Next Generation Mobile Broadcasting, CRC Press, 2013.

[17] D. Vargas, D. Gozálvez, D. Gómez-Barquero, and N. Cardona, "MIMO for DVB-NGH, The Next Generation Mobile TV Broadcasting," IEEE Communications Magazine, vol. 57, no. 7, pp. 130-137, 2013.

[18] J. J. Giménez, D. Gómez-Barquero, S. Bergsmark and E. Stare, “Time Frequency Slicing for Future Digital Terrestrial Broadcasting Networks," IEEE Trans. on Broadcasting, vol. 60, no. 1, pp. xyz-abc, 2014.

[19] EBU Technical Report 3348, "Frequency and Network Planning Aspects of DVB-T2,” Nov. 2013.

[20] J. J. Giménez, D. Gozálvez, D. Gómez-Barquero, and N. Cardona, “A Statistical Model of the Signal Strength Imbalance between RF Channels in a DTT Network," IET Electronic Letters, vol. 48, no. 12, pp. 731-732, June 2012.

[21] D. Gozálvez, J. J. Giménez, D. Gómez-Barquero, and N. Cardona, "Rotated Constellations for Improved Time and Frequency Diversity in DVB-NGH,” IEEE Trans. on Broadcasting, vol. 59, no. 2, pp. 298-305, 2013.

[22] T. Jokela, M. Tupala, and J. Paavola, "Analysis of Physical Layer Signaling Transmission in DVB-T2 Systems," IEEE Trans. on Broadcasting, vol. 56, no. 3, pp. 410-417, Sept. 2010.

[23] C. Bormann, et al., "RObust Header Compression (ROHC): Framework and Four Profiles: RTP, UDP, ESP, and Uncompressed," ITEF RFC 3095, July 2001.

[24] H. Jiang and P. A. Wilford, "A Hierarchical Modulation for Upgrading Digital Broadcast Systems,” IEEE Trans. Broadcasting, vol. 51, no. 2, pp. 223-229, Jun. 2005.

[25] J. Qi, J. Robert, K. L. Chee, M. Slimani, and J. Zoellner, "DVB-T2 MISO Field Measurements and a Calibrated Coverage Gain Predictor," Proc. IEEE BMSB, Seoul, South Korea, 2012.

[26] S. Kaiser, "Spatial Transmit Diversity Techniques for Broadband OFDM Systems,” Proc. IEEE GLOBECOM, San Francisco, USA, 2000.

[27] D. Gómez-Barquero, P. F. Gómez, D. Gozálvez, B. Sayadi, and L. Roullet, "A Novel Physical Layer Split FEC Scheme for Long Time Interleaving With Fast Zapping Support," IEEE Trans. on Broadcasting, vol. 58, no. 2, 269-276, 2012.

[28] D. Gozálvez, J. J. Giménez, D. Gómez-Barquero, and N. Cardona, “Combined Time, Frequency and Space Diversity in DVB-NGH," IEEE Trans. on Broadcasting, vol. 59, no. 4, pp. 674-684, 2013.

David Gómez-Barquero received the double M.Sc. degree in telecommunications engineering from the Universitat Politècnica de València (UPV), Spain, and the University of Gävle, Sweden, in 2004, and the Ph.D. degree in telecommunications from UPV in 2009. During his doctoral studies, he was a Guest Researcher at the Royal Institute of Technology, Sweden, the University of Turku, Finland, and the Technical University of Braunschweig, Germany. He also did an internship at Ericsson Eurolab, Germany. From 2010 to 2011, he was a Post-Doctoral Guest Researcher at the Fraunhofer Heinrich Hertz Institute, Germany.

He is currently a Senior Researcher (Ramon y Cajal Fellow) at the Institute of Telecommunications and Multimedia Applications (iTEAM), UPV, where he leads a research group working on multimedia broadcasting, in particular on the optimization of 3GPP multimedia broadcast multicast services (MBMS) and digital video broadcasting (DVB) systems.

Since 2008, he has been actively participating in the European digital television standardization forum DVB. He participated in the validation of DVB-T2, and in the standardization processes of its mobile profile T2-Lite and its handheld evolution DVB-NGH. He also contributed to the DVB-T2 implementation guidelines, and co-edited the implementation guidelines for upper layer forward error correction. He was very involved in the promotion and adoption of DVB-T2 in Colombia, and since the second half of 2012 he has been working with the spectrum regulator on the spectrum and network planning and optimization of DVB-T2. Since then, he is also a Visiting Professor at the Sergio Arboleda University of Bogota, Colombia.

Dr. Gómez-Barquero is the Editor of the book "Next Generation Mobile Broadcasting" (CRC Press), and the Vice-Chairman of the Modulation and Coding Ad-Hoc Group of the ATSC 3.0 standardization process.

Catherine Douillard received the engineering degree in telecommunications from the Ecole Nationale Supérieure des Télécommunications de Bretagne, France, in 1988, the Ph.D. degree in 
electrical engineering from the University of Western Brittany, France, in 1992, and the accreditation to supervise research from the University of Southern Brittany, France, in 2004.

She is currently a Professor in the Electronics Department of Telecom Bretagne where she is in charge of the Algorithm-Silicon Interaction research team. Her main research interests are turbo codes and iterative decoding, iterative detection, the efficient combination of high spectral efficiency modulation and turbo coding schemes, diversity techniques and turbo processing for multi-carrier, multi-antenna and multiple access transmission systems. In 2009, she received the SEE/IEEE Glavieux Award for her contribution to standards and related industrial impact.

Peter Moss joined the BBC in 1980 after gaining a BA in Physics from the University of Oxford, UK. He is currently a lead research engineer at the BBC's Research and Development department in West London. Initially involved in the design of transmitters and receivers for broadcast and ancillary services, he was responsible for the 175W PA design used in the first 27 DAB broadcast transmitters. He worked in the late 1990s on a wireless television camera based on the DVB-T standard where he was principal RF architect and circuit designer. More recently he has led the team working on On-Channel Repeaters for DVB-T, DVB-T2 and DAB. In 2006 he chaired an industry consortium demonstrating the real-world capabilities of cross-polar broadcast MIMO. He was Task Force Chair for MIMO in the DVB-NGH group developing a next-generation transmission standard for handheld devices. Currently he is continuing his work on the possibilities of MIMO for broadcast to fixed receivers and is leading the team which creating a MIMO radio-camera for $4 \mathrm{k}$ video based on the NGH 4 x 2 hybrid MIMO scheme.

Vittoria Mignone received the "Laurea in Ingegneria Elettronica" degree from Politecnico di Torino, Turin, Italy in 1990.

She has been with the RAI Research Centre since 1992, involved in the studies for the definition of the ETSI Standards for digital television by satellite, cable and terrestrial channels. Her activities are in the field of advanced digital modulation and channel coding techniques for satellite and terrestrial transmissions. She is author of patents and technical papers. 